\title{
Cryptanalysis to Secure System
}

\author{
Banita Chadha \\ Research Scholar \\ Galaxy Global Imperial Technical campus \\ Computer Science Department Kurukshetra \\ University \\ India
}

\author{
Puneet Goswami, Ph.D \\ Galaxy Global Group Of Institutions \\ Computer Science Department \\ Head Of Department Kurukshetra University \\ India
}

\begin{abstract}
In this paper we describe how to implement 3D password. As it is free from textual password as well as graphical password. Hence it is free from attackers. 3D password is used to overcome the drawbacks of previously existing security or authentication schemes. 3D password is well created by user itself by clicking on number the images which are displayed on desktop, By this it will overcome the textual password as well as graphical password. 3D password is based on sequence of clicks which are made by the user during his login. Hence it deals with pattern as well as interaction made by user itself.
\end{abstract}

\section{General Terms}

Pattern recognition, Interaction by user, Convex Hull Clicking

\section{Keywords}

3D Password system Design, Convex Hull Click Scheme, Conlusion.

\section{INTRODUCTION}

Authentication is just the begning for protecting any resource or any system. As with the rapid growth of Internet there is highest probability for hackers to attack the system by use of textual password or may be graphical password. To overcome such situation 4 authentication techniques are there:
1) Knowledge Based
2) Token Based
3) Biometrics
4) Recognition Based.

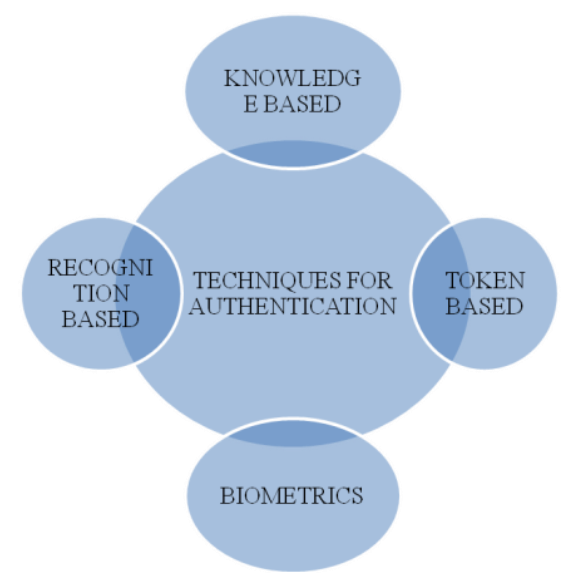

But in case of computer there are only two techniques for authentication:

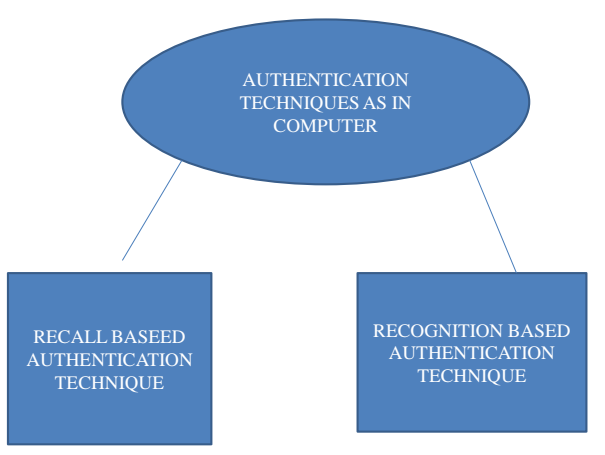

1) Recall Based 2) Recognition Based

Recall Based and recognition based are much more similar but they differentiate only by there password. Recall based authentication technique based on textual password and in this user requires to repeat password which user created earlier.

Recognition Based is used in graphical password so, it is secure from recall based technique but not as much as $3 \mathrm{D}$ password.

\section{3D PASSWORD SYSTEM DESIGN}

To built 3D password the user must be familiar with 3D environment which reflects the requirements for security of any resource or system on whch user wants to create.

For 3D password sysem design 3 major steps are required:

1) User may first fill the login form which use textual password. User must enter his textual password which is already created by user itself. Such password include the recall based authentication technique.

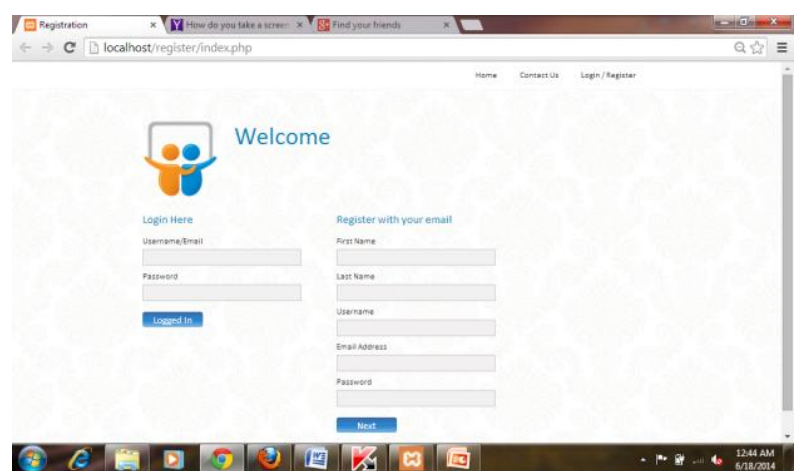


2) After recall based authentication technique user will enter graphical password. This graphical based technique deals with clicking on the pass-points or images which get displayed on to the desktop.

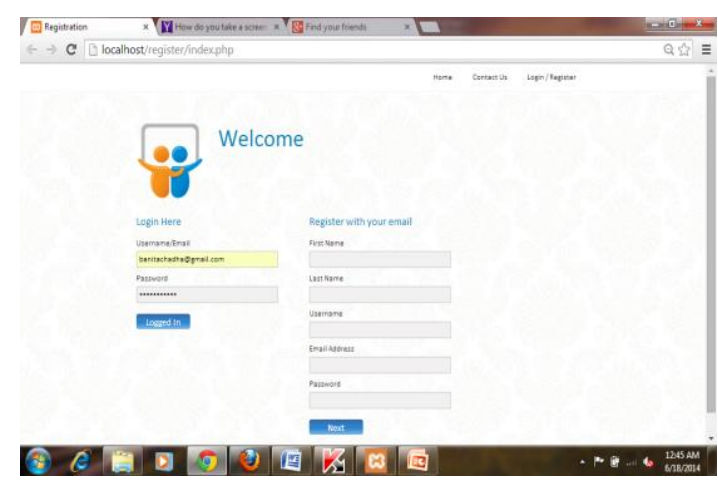

3) This 3D password have a sequence of clicking on pass-points which is created by the user.

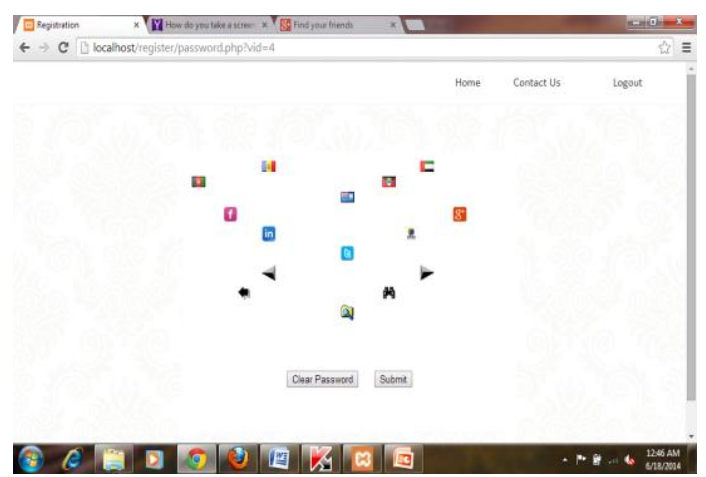

\section{CONVEX HULL CLICK SCHEME}

Convex Hull Click scheme used to make system more secure. This method deals with clicking on passpoints and that passpoints which are clcked by user is not more than 8 . Thus if

$$
\operatorname{Lmax}=8
$$

That means user click 8 images for its 3D password. Here Lmax is 8.i.e. maximum length for password is 8 or less tah 8 but not more than 8 .

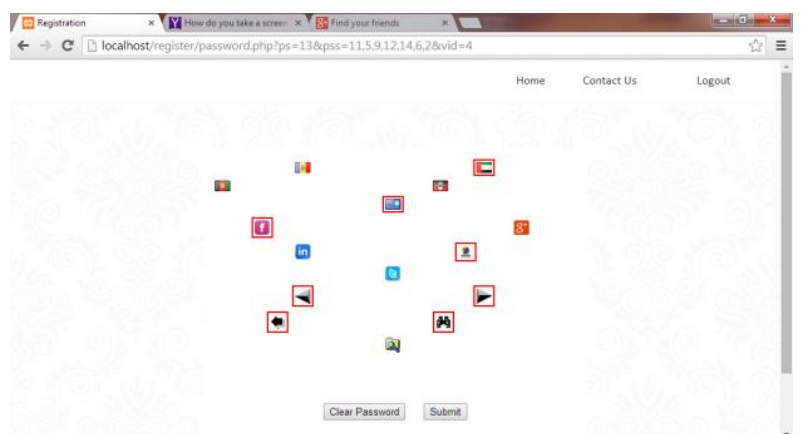

\section{(2) E D}

The very first step is how to create convex hull . It is the area which is closed is such a pattern that inside that area there are number of images present. This closed area is created by the user after clicking on 8 or less than 8 pass points or images which are displayed on desktop.

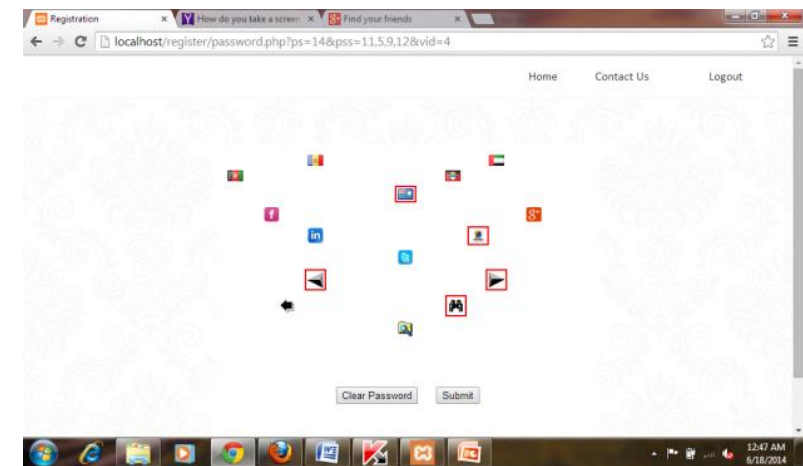

Similarly if $\operatorname{Lmax}=4$ that means the user clicked only 4 images in such a pattern that hull is created Thus length of password is directly proportional to the security.

After creating hull user will click on various passpoints inside that hull in a particular pattern which get remembered by user. Sequence of clicking on various pass points is the password of that user. Hence it is free from Shoulder surfering attack. No attacker will judge the password as it is created by number of clicks.

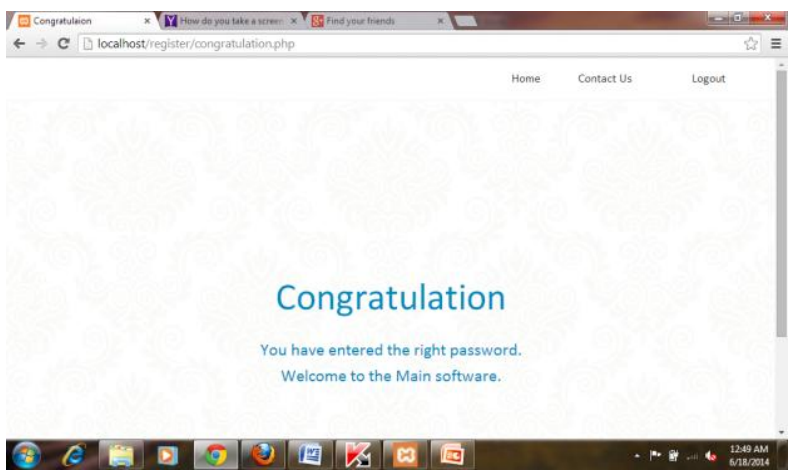

\section{CONCLUSION}

The conclusion of my research is that 3D password will overcome textual password as well as graphical password. 3D password must be used in the case of any confidential work such that sender need not to be worried about the hacking system. The main thing is the common key which must be known to sender and receiver. Convex Hull Click Scheme is easy to use and is secure enough because it is based on the pattern matching which is created by user only.

\section{REFERENCES}

[1] Vishal Kolhe, VipulGunjal, SayaliKalasakar, PranjalRathod, " Secure Authentication with 3D Password", International Journal of Engineering Science and Innovtive Technology(IJESIT), Volume 2, Issue 2, March 2013.

[2] Mr.Jaywant N. Khedkar, Ms.Pragati P. Katalkar, Ms.Shalini V. Pathak, Mrs.RohiniV.Agawane, "Integration of Sound Signature in 3D PasswordAuthentication System",International Journal of Innovative Research in Computer and Communication Engineering,Vol. 1, Issue 2, April 2013.

[3] A Aswathy Nair, Theresa Rani Joseph, Jenny Maria Johny," A Proficient Multilevel Graphical Authentication System", Interanational Journal of Science, Engineering, and Technology Research (IJSETR), Volume 2, No 6, June 2013. 
[4] R.N.Muneshwar, S.K.Sonkar, "Virtual Environments Provide Mammoth Security for Critical Server", International Journal of Engineering and Advanced Technology (IJEAT), Volume-2, Issue-3, February 2013.

[5]ShubhamBhardwaj, Varun Gandhi, VarshaYadav, LalitPoddar, "New Era of Authentication: 3-D Password", International Journal of Science, Engineering and Technology Research (IJSETR), Volume-1, Issue-5, November 2012.

[6] A.B.Gadicha , V.B.Gadicha , "Virtual Realization using 3D Password", International Journal of Electronics and Computer Science Engineering, ISSN 2277-1956/V1N2216-222.

[7] Grover Aman, Narang Winnie,"4-D Password: Strengthening the Authentication Scene", International
Journal of Scientific \& Engineering Research, Volume 3, Issue 10, October-2012.

[8]Mr. Namdev A. Anwat,Mr. Dattatray S. Shingate,Dr. Varsha H. Patil, "A Secure Authentication Mechanism using 3D Password", International Journal of Advance Research in Science, Engineering and Technology, Vol.01, Issue 01, pp. 29-37.

[9]Fawaz A. Alsulaiman ,Abdulmotaleb El Saddik,"Three Dimensional Password for More Secure Authentication", IEEE Transactions on Instrumentation and Measurement, Vol. 57, No. 9, September 2008.

[10]Harshil Shah, ChiragLakhani, SagarHaldankar,"Graphical Password Authentication Based on Polygon Visualization",International Journal of Engineering Research and Applications (IJERA) ISSN: 2248-9622. 